International Journal of Applied Mathematical Research, $5(2)(2016) 117-122$
International Journal of Applied Mathematical Research
WPC
Website: www.sciencepubco.com/index.php/IJAMR
doi: $14419 /$ ijamr.v5i2.6033
Research paper

\title{
Lie group and RK4 for solving nonlinear first order ODEs
}

\author{
Sami H. Altoum ${ }^{1 *}$ \\ ${ }^{1}$ Umm Al-Qura University, Al-Qunfudah Mathematics department, Macca, KSA \\ **orresponding author E-mail: shtoum@uqu.edu.sa
}

\begin{abstract}
This paper deals with a numerical comparison between Lie group method and RK4 for solving an nonlinear ordinary differential equation. The Lie group method will be introduced as a analytical method and then compared to RK4 as a numerical method. Some examples will be considered and the global error we be computed numerically.
\end{abstract}

Keywords: Lie group, Symmetry group, Rung-Kutta method, Numerical solution.

\section{Introduction}

Consider the first order ordinary differential equations of the form

$y^{\prime}(x)=f(x, y), \quad y\left(x_{0}\right)=y_{0}$

where $f$ is given and $y$ is an unknown function of $x$. When $f$ is continuous over some interval, on can found the general solution using different methods.

Such problem often arise in science fields. Ordinary differential equations frequently occur as mathematical models in many branches of science, engineering and economy. Unfortunately it is seldom that these equations have solutions that can be expressed in closed form, so it is common to seek approximate solutions by means of numerical methods; nowadays this can usually be achieved very inexpensively to high accuracy and with a reliable bound on the error between the analytical solution and its numerical approximation. We present some computational methods to approximate the solution of ordinary differential equations (ODEs). In [6],[11] and [4] there is many methods stability of linear and nonlinear equation. Euler's algorithm is not very accurate

and also suffers from instabilities. The mathematicians Runge and Kutta [5] discovered a class of algorithms that are based on making multiple Euler steps. These Runge-Kutta algorithms [9] have been found to work very well in many applications. Phohom Siri and Lidwadia [12] solve the problem using the accelered of Rung-Kutta method. Rabieiaud Ismail [13] proposed the third-order improved Rung-Kutta method for solving 1, other methods also was investigated to solve the first order ODE like Lie group method. The Lie group is a differential manifold that the group operations are compatible with the smooth structure, [1]. Moreover, Lie gave a classification of ODEs in terms of their symmetry group which is a rigid mapping from an object to itself or another object. Fortunately, the properties of symmetries provide a unique tool for solving differential equations. It must preserve the structural properties of the original coordinate. The analytical solution of ODEs will be computed using Lie group if it's possible then compared to the other one obtained using RK4 [8]. The comparison will be tested in the case of nonlinear ODEs [11]. We will consider in our test only a first order differential equation and the numerical solution to secondorder and higher-order differential equations is formulated in the same way as it is for first-order equations. We have attempted to give a some examples to the use of Lie group methods for the solution of first-order ODEs. The Lie group method of solving higher order ODEs [10] and systems of differential equations is more involved, but the basic idea is the same: we find a coordinate system in which the equations are simpler and exploit this simplification. A state variable will be introduced for the output and each of its first $n-1$ derivatives (for an $n^{\text {th }}$-order differential equation). The numerical integration algorithm is applied to each of the state variables. The outline of the paper is the following: In Section 2, we introduce the Lie group method used to solve the ODEs.

In section 3 a rapid review of Runge Kutta 4 is presented and some practical examples to compare the two methods used to solve an ODEs. In section 4, we solve a second order odes as an application. Finally, we conclude.

\section{Lie group}

Let $(r(x, y), s(x, y))$ be the canonical coordinates and a given differential equation becomes separable, for the simplest cases we obtain either $\frac{d s}{d r}=f(r)$ or $\frac{d s}{d r}=f(s)$. For the sake of definiteness, we treat only the case $\frac{d s}{d r}=f(r)$. The $(R, S)=(r, s+\lambda)$, that is, in the new coordinates there is a point symmetry, [3]

$P_{\lambda}:(r, s) \mapsto(R, S)=(r, s+\lambda)$

that amounts to translation in the $s$-direction. Then the tangent vector at $(r, s)$ will be

$\left.\frac{d R}{d \lambda}\right|_{\lambda=0}=0$
$\left.\frac{d S}{d \lambda}\right|_{\lambda=0}=1$ 
Taking derivatives with respect to $\lambda$ at $\lambda=\lambda_{0}$ leads to

$$
\begin{aligned}
\left.\frac{d R}{d \lambda}\right|_{\lambda=\lambda_{0}} & =\left.\frac{d R}{d x} \frac{d x}{d \lambda}\right|_{\lambda=\lambda_{0}}+\left.\frac{d R}{d y} \frac{d y}{d \lambda}\right|_{\lambda=\lambda_{0}} \\
& =\frac{d r}{d x} \xi+\frac{d r}{d y} \eta \\
& =0 \\
\left.\frac{d S}{d \lambda}\right|_{\lambda=\lambda_{0}} & =\left.\frac{d S}{d x} \frac{d x}{d \lambda}\right|_{\lambda=\lambda_{0}}+\left.\frac{d S}{d y} \frac{d y}{d \lambda}\right|_{\lambda=\lambda_{0}} \\
& =\frac{d s}{d x} \xi+\frac{d s}{d y} \eta \\
& =\frac{d s}{d x} \xi+\frac{d s}{d y} \eta \\
& =1
\end{aligned}
$$

Using

$r_{x} \xi+r_{y} \eta=0, \quad s_{x} \xi+s_{y} \eta=1$

An essential feature of a symmetry group of ODEs is that it conserves the set of solutions of the differential equation admitting this group[7]. Namely, the symmetry transformations merely permute the integral curves among themselves.Such integral curves are termed invariant solutions [2]. Equation 5 represent a system of ODEs equations with the variable $r=r(x ; y)$ and $s=s(x, y)$; whose solutions are $r=c$ and $s=k$ are first integrals in fact the general order ordinary differential equation admitting the group of translations along the $x$-axis. Moreover, any one parameter group reduces in proper variables to the group of translations. These new variables, canonical variables $r(x, y) ; s(x, y)$ obtained by solving the equation. In particular, any first-order equation with a known one-parameter symmetry group can be integrated by quadrature using canonical variables. Integration of second order equations requires two independent infinitesimal symmetries (namely two dimensional Lie algebras). To solve the ode we do the following:

Get the Lie symmetries of unknown solutions by using symmetry condition [2]

$\eta_{x}-\xi_{y} h^{2}+\left(\eta_{y}-\xi_{x}\right) h-\left(\xi h_{x}+\eta h_{y}\right)=0$

and use the solution to linearized symmetry condition to find a coordinate system $(r ; s)$ in which the solutions depend on only one of the variables, before final step we substitute the canonical coordinates in to

$\frac{d s}{d r}=\frac{s_{x}+h(x, y) s_{y}}{r_{x}+h(x, y) r_{y}}$

and solve the differential equation in the coordinate system. Finally express the solution in the original coordinates, see [3].

\section{Numerical method}

We shall consider the solution of sets of first-order differential equations only. Users interested in solving higher order ODES can reduce their problem to a set of first-order equations. Fortunately, the method of Lie group gives sometimes the solution of ODEs, but it's difficult to obtain an exact solution. For that, we will use numerical methods to obtain a solution. Since the numerical method used to compute an approximation at each step of the sequence, errors are compounded at every step. we introduce the numerical method will be compared to Lie group method and we don't forget the comparison of the error at each example will be presented.
In this context, we will use Runge Kutta-4 method which is the most widely one-step methods to solve

$\frac{d}{d x} y(x)=f(x, y(x))$

involves defining 4 quantities $k_{n}$ as follows:

$k_{n}=f\left(x_{i}+c_{n} h, y_{i}+h \sum_{m=1}^{M} \alpha_{n, m} k_{m}\right)$

here, $y_{i} \sim y\left(x_{i}\right)$ represents our numeric approximation to solution of the differential equation at $x=x_{i}$. The coefficients $c_{n}$ and $\alpha_{n, m}$ are constants that we are going to choose shortly, and $h=x_{i+1}-x_{i}$ is the stepsize. Once one calculates $k_{n}$ given at $\left(x_{i}, y_{i}\right)$ the value of $y_{i+1} \sim y\left(x_{i+1}\right)$ is given by

$y_{i+1}=y_{i}+h \sum_{n=1}^{M} b_{n} k_{n}$

In this expression, the $b_{n}$ are constants.

$k_{1}=f\left(x_{i}, y_{i}\right)$

$k_{2}=f\left(x_{i}+1 / 2 h, y_{i}+1 / 2 h k_{1}\right)$

$k_{3}=f\left(x_{i}+1 / 2 h, y_{i}+1 / 2 h k_{2}\right)$

$k_{4}=f\left(x_{i}+h, y_{i}+h k_{3}\right)$

then we have

$y_{i+1}=y_{i}+h\left(1 / 6 k_{1}+1 / 3 k_{2}+1 / 3 k_{3}+1 / 6 k_{4}\right)$

We recall that using $M=4$ (RK4) the global error is $O\left(h^{4}\right)$.

\section{Example 1}

As a first easy example, that can be solved using Lie group and RK4. The Bernoulli equation

$\frac{d y}{d x}=y+y^{-1} e^{x}$

when substituted into condition 6 , leads to

$\eta_{x}-\xi_{y}\left(y+y^{-1} e^{x}\right)^{2}+\left(\eta_{y}-\xi_{x}\right)\left(y+y^{-1} e^{x}\right)-\left(\xi\left(y^{-1} e^{x}\right)+\eta\left(1-\frac{e^{x}}{y^{2}}\right)\right)=0$

This is, again, is too difficult as it sits, so we try a few simplifying assumptions before we discover that $\xi=1, \eta=\eta(y)$ yields

$\eta_{y}\left(y+y^{-1} e^{x}\right)-\left(y^{-1} e^{x}\right)-\eta\left(1-y^{-2} e^{x}\right)$.

Because some terms depend only $y$, we solve $y \eta_{y}-\eta=0$ to obtain $\eta=c y$. Inserting this form of $\eta$ into the remaining equation $\eta_{y}+$ $y^{-1} \eta-1=0$, we arrive at $\eta=\frac{y}{2}$. Now that we have settled on the symbols $(\xi, \eta)=\left(1, \frac{y}{2}\right)$, we find canonical coordinates by solving $\frac{d y}{d x}=\frac{\eta}{\xi}=\frac{y}{2}$, to get $r$, so $r=c=y e^{-\frac{x}{2}}$. The second coordinate $s$ is found by integrating $d s=\frac{d x}{1}$ to get $s=x$. The next step is to find the differential equation in the canonical coordinates by calculating

$\frac{d s}{d r}=\frac{s_{x}+s_{y} h}{r_{x}+r_{y} h}$.

We learn that

$\frac{d s}{d r}=\frac{1}{-\frac{1}{2} y e^{-\frac{x}{2}}+e^{\frac{-x}{2}}\left(y+y^{-1} e^{x}\right)}=\frac{1}{\frac{1}{2} y e^{-\frac{x}{2}}+y^{-1} e^{\frac{x}{2}}}$

Expressing $\frac{1}{2} y e^{\frac{-x}{2}}+y^{-1} e^{\frac{x}{2}}$ in terms of $r$ and $s$ leads to $\frac{r}{2}+\frac{1}{r}$, whence $\frac{d s}{d r}=\frac{r}{\frac{r^{2}}{2}+1}$. 
This integrating to

$s=\ln \left(\frac{r^{2}}{2}+1\right)+c$.

Returning to the original coordinates, we obtain

$y= \pm \sqrt{c e^{2 x}-2 e^{x}}$

Here, we compare the solution of the Bernoulli equation obtained using Lie group and the numerical solution given by RK4, see Figure ??. We see for even a moderate number of steps, the agreement between the Runge-Kutta method and the analytic solution is remarkable. We can quantify just how much better the Runge-Kutta method does by defining a measure of the global error $\varepsilon$ as the magnitude of the discrepancy between the numerical and actual values of $y(1)$, see Figure ??.

Clearly, the error for the Runge-Kutta method is several orders of magnitude lower. Furthermore, the global error curves both look linear on the log-log plot, which suggests that there is a power law dependence of $\varepsilon$ on $N$. We can determine the power $a$ by fitting a power law to the data obtained in our Maple code this leads that the global error using RK4 is $O\left(h^{3.9735070743}\right)$ this match our expectation that the one step error is $O\left(h^{5}\right)$.

\section{Example 2}

We consider here the case where

$y^{\prime}=\frac{y}{x-y}, y(0)=2$.

Using the same technique of Lie group presented in the first example, we have the following steps:

$\left(y^{2}-2 y x+x^{2}\right) \eta_{x}+\left(y x-y^{2}\right) \eta_{y}+\left(y^{2}-y x\right) \xi_{x}-y^{2} \xi_{y}+y \xi-x \eta=0$, we can simplify to

$\left(y^{2}-2 y x+x^{2}\right) \eta_{x}+\left(y x-y^{2}\right) \eta_{y}+\left(x y-y^{2}\right) \eta_{y}-x \eta-y^{2} \xi_{y}+y \xi=0$

if we choose $\xi$ to be function of $y$ alone. Because of the independence of $\xi$ upon $y, y \xi-y^{2} \xi_{y}=0$ separately, and we can solve this to obtain $\xi=y$. Now, the remainder in $\eta$ is difficult to solve but is identically satisfied by $\eta=0$. Therefore, we try $(\xi, \eta)=(y, 0)$. Integrating the characteristic equation, we get $(r, s)=\left(y, \frac{x}{y}\right)$. Calculate

$\frac{d s}{d r}=\frac{s_{x}+s_{y} y^{\prime}}{r_{x}+r_{y} y^{\prime}}$

we see that $\frac{d s}{d r}=\frac{-1}{r}$, which upon substitution of the original variables becomes

$\frac{x}{y}=-\ln y+c$.

As we see, we have not an explicit solution, so in this example using the Lie group technique one can not be able to determine the solution explicitly. In other word, it's better to use a numerical method to simplify the work. Once again, we will test the RK4, in this second example. First, let us recall that the analytical solution of this example is given using the Lambert $W$ function. The Lambert W function satisfies

LambertW $(x) \mathrm{e}^{\operatorname{Lambert} W(x)}=x$

and using Maple one can determine the analytical solution and it's given by

$y(x)=\mathrm{e}^{\operatorname{Lambert} W(-1 / 2 x)+\ln (2)}$

Lie group and RK4 will be used to solve 7, Figure 1(a)shows the analytical and numerical solution. We see for even a moderate

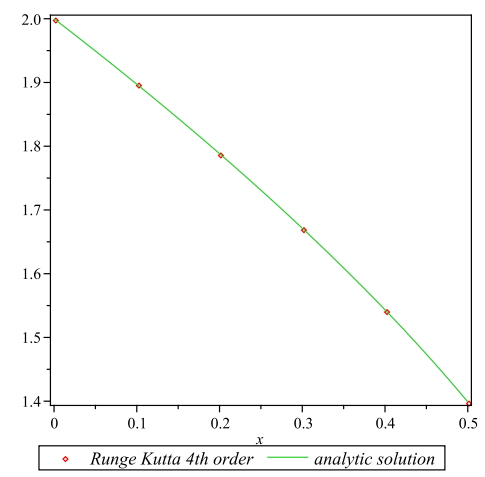

(a) Analytic solution (Lie group) and numerical solution

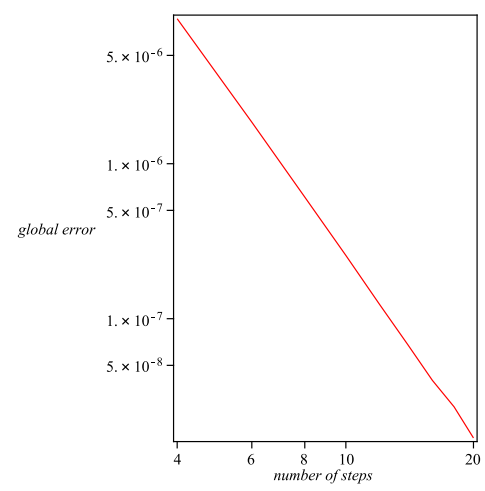

(b) Error between analytic solution and RK4

Figure 1: Solution and error of first order ode

number of steps, the agreement between the Runge-Kutta method and the analytic solution is remarkable. We can quantify just how much better the Runge-Kutta method does by defining a measure of the global error $\varepsilon$ as the magnitude of the discrepancy between the numerical and actual values of $y(0.5)$, see Figure 1(b). Once again, the global error using RK4 is $O\left(h^{3.8711520000}\right)$ this match our expectation that the one step error is $O\left(h^{5}\right)$. In this example, we work in the interval $[0,0.5]$ to avoid any singularities can be found.

\section{Example 3}

Consider the equation

$\frac{d y}{d x}=\frac{y}{x}+x$

Substitute the equation into the linearized symmetry condition 6 , to get

$\eta_{x}-\xi_{y}\left(\frac{y}{x}+x\right)^{2}+\left(\eta_{y}-\xi_{x}\right)\left(\frac{y}{x}+x\right)-\left(\xi\left(1-\frac{y}{x^{2}}\right)+\eta\left(\frac{1}{x}\right)\right)=0$

It is necessary to solve this equation for $\xi$ and $\eta$. In its current form, this is a very difficult task. Therefore, we suppose that $\xi=0$ and $\eta$ is a function in $x$ only. Then we get

$\eta_{x}=\frac{\eta}{x}$

which we can easily solved to get $\eta=c x$. Now we can find the canonical coordinates $r$ and $s$ when the $\xi(x, y)=0, r=x$. To find $\mathrm{s}$ we can solve

$d s=\frac{d y}{\eta}$. 
Therefore

$s=\frac{y}{c x}$.

Now set $c=1$ to get

$(r(x, y), s(x, y))=\left(x, \frac{y}{x}\right)$

and

$s_{x}=\frac{-y}{x^{2}}, s_{y}=\frac{1}{x}$.

Now we can substitute $r$ and $s$ into $\frac{d s}{d r}=\frac{s_{x}+h(x, y) s_{y}}{r_{x}+h(x, y) r_{y}}$, to get

$\frac{d s}{d r}=\frac{\frac{-y}{x^{2}}+\left(\frac{y}{x}+x\right) \frac{1}{x}}{1+0}=1$

Therefore $s=r+k$, where $k$ is constant. Substituting $x$ and $y$ bach in we get

$\frac{y}{x}=x+k$.

The general solution of equation is $y=x^{2}+k x$. Figure 2(a) compares the numerical solution and the analytic solution obtained using RK4 The global error computed at $x=1$ when the number of steps $N$ change, see figure 2(b) and the error is $O\left(h^{3.4355839580}\right)$ this match our expectation that the one step error ought to be $O\left(h^{5}\right)$. In this example, we work in the interval $[0.1,1]$ to avoid the singularities at $x=0$.

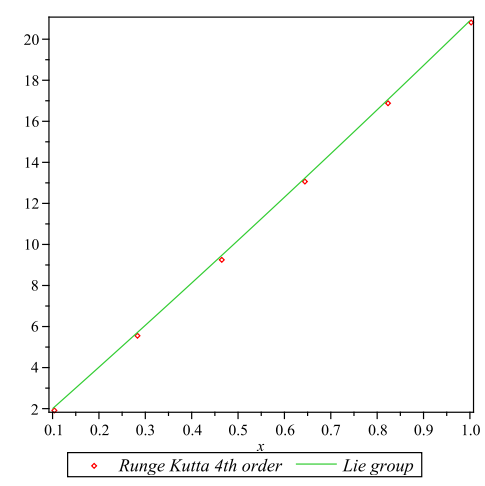

(a) Analytic solution (Lie group) and numerical solution (RK4)

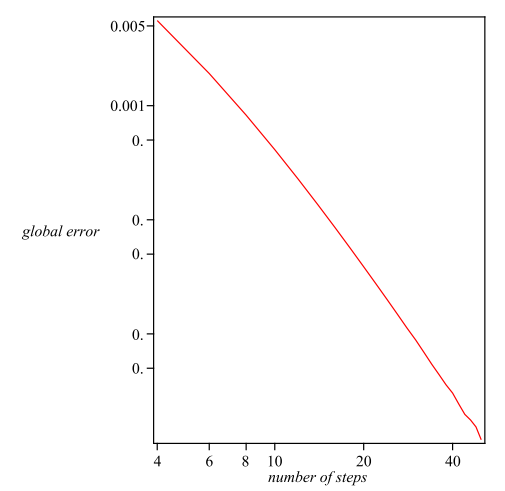

(b) Error between analytic solution and RK4

Figure 2: Solution and error of first order ode

\section{Example 4}

Let us consider this example $y^{\prime}=100 x^{2}+x-100 y, x \in[0,1], y(0)=$ 2 . This example was considered by many authors. The analytical solution obtained using Lie group is

$y=x^{2}+2 \mathrm{e}^{-100 x}$

MATLAB has a number of tools for numerically solving ordinary differential equations. We will focus on ode 45 which implement versions of Runge-Kutta 4-5th-order. Remember that, MATLAB has two Runge-Kutta methods available, ODE23 and ODE45. Each of them uses a variable time step rather than a constant value of $h$. The program chooses its own value to use for $h$ at each timestep in order to keep its estimation of the error small. we will numerically approximate the solution of the first order differential equation. It is important to point out here that MATLAB continues to use roughly the same partition of values that it originally chose; the only thing that has changed is the values at which it is printing a solution. In this way, no accuracy is lost, and the solution is presented in Figure 3. In this example the classical RK4 can not be used, and the there is non convergence to the exact solution and exactly we have a jump using RK4, see Figure 3(b).

\section{Second order of ODE.}

Fortunately, the second order odes can be reduced to a first order odes then following the previous steps we present a comparison between the Lie group technique and RK4 to solve a second order ODEs. For this purpose and to illustrate our strategy of comparison we consider only one example. The RK4 and forward Euler method will be compared to Lie group technique. Figure ?? give the behavior of the solution using RK4, Forward Euler and Lie group technique. We notice that there is a big error occurs using forward Euler. Moreover Figure ?? confirm our predictions that there is a small error between analytical and numerical solution using RK4 but there is no-convergence when one use Forward Euler method.

\section{Example 5}

$y^{\prime \prime}+F(x) \hat{y}+G(x) y=0$

A transformation of the form $A_{t}:(x, y) \mapsto(x, f(t) y)$ is a symmetry of (5). The orbits of $A_{t}$ are again vertical lines so we take $r=x$. Additionally $\varepsilon=0$ and 5 is $s_{y} \eta=1=s_{y}\left(f^{\prime}(0) y\right)$. Now let $f(t)=e^{t}$, so that

$s_{y}=\frac{1}{f^{\prime}(0) y}=\frac{1}{y}$.

This has $s=\ln y$ as a solution which is invertible by $y=e^{s}$. Then we have

$y^{\prime}=e^{s} s_{x}$

$y^{\prime \prime}=e^{s} s_{x}^{2}+e^{s} s_{x x}=e^{s}\left(s_{x x}+s_{x}^{2}\right)$

so 8 becomes

$s_{x x}+s_{x}^{2}+F(x) s_{x}+G(x)=0$.

9 Since $s$ does not appear explicitly in the transformed equation we can make the substitution $z=s_{x}$ so that (6) becomes

$z_{x}+z^{2}+F(x) z+G(x)=0$ 

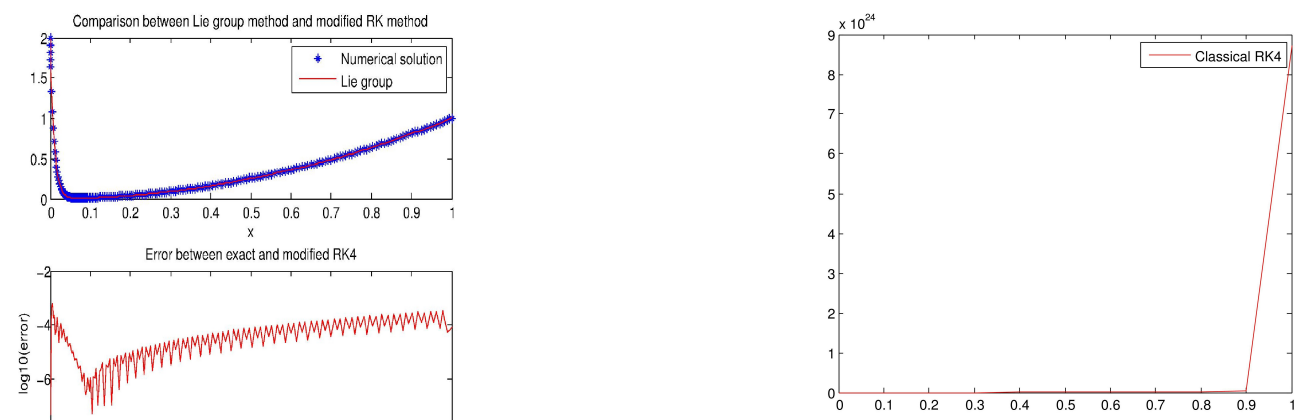

(a) Comparison between Lie group and numerical solution

(b) Jump in the solution using classical RK4

Figure 3: Comparison between Lie group and numerical solution and Jump in the solution using classical RK4

which is a first order ODE. Now let $F(x)=1$ and $G(x)=x$

$\frac{d}{d x} y(x)=-(y(x))^{2}-y(x)-x$

and the exact solution is given by $y(x)$ where $y(x)$ is fractional function equal the following:

$$
\begin{gathered}
\text { Num }=\frac{(5 B i(1 / 4)+2 B i(1,1 / 4)) A i(-x+1 / 4)}{5 A i(1 / 4)+2 A i(1,1 / 4)}+2 \frac{(5 B i(1 / 4)+2 B i(1,1 / 4)) A i(1,-x+}{5 A i(1 / 4)+2 A i(1,1 / 4)} \\
-B i(-x+1 / 4)-2 B i(1,-x+1 / 4)
\end{gathered}
$$

deno $=-2 \frac{(5 B i(1 / 4)+2 B i(1,1 / 4)) A i(-x+1 / 4)}{5 A i(1 / 4)+2 A i(1,1 / 4)}+2 B i(-x+1 / 4)$

The Airy $A i$ and $B i$ functions. If we compare and contrast two of the most well known techniques for the solving our second order ODE. The Runge-Kutta method is very similar to Euler's method except that the Runge-Kutta method employs the use of parabolas 2nd order and quadric curves 4 th order to achieve the approximations. For small $N$ the Euler's method seem to not converge ti the exact solution and this method method need more point to achieve the accuracy instead of RK4 which converge to the exact solution for small $N$. The four following figures show the curves for different value of $N$.

\section{Conclusion}

In this paper we have investigated the solution of ODEs using two approaches. The first one, is the Lie group and the second one is numerical method based on RK4. Using Lie group, one can not determine an explicit solution of an ODEs in general case, so the numerical method like RK4, is a good technique helped us to find an approximation of the exact solution with small error. In the last, we note that the Euler methods are tested numerically and we have not convergence and the determined global error is about $O\left(h^{9467945525}\right)$ and this don't match our expectation that if the one-step error is $O\left(h^{2}\right)$. Euler's algorithm is not very accurate and also suffers from instabilities. Considering the results obtained in this paper, we plan in the future to tackle the following questions:

- We will compare the Lie group method and the Runge Kutta Nystrom (RKN) method.

- We solve a system of second order ode using Lie group and different stages-RKN.

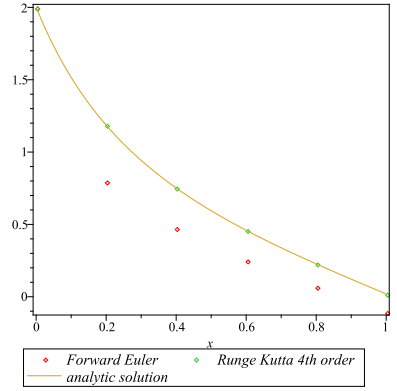

(a) Case $\mathrm{N}=5$

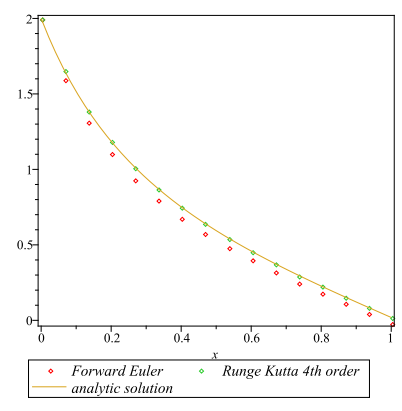

(c) Case $\mathrm{N}=15$

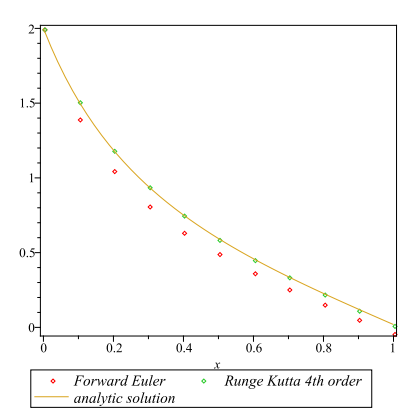

(b) Case $\mathrm{N}=10$

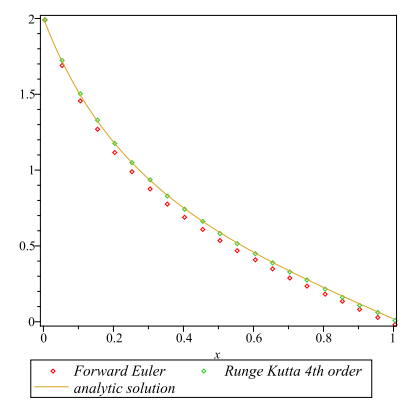

(d) case $\mathrm{N}=20$
Figure 4: Behavior of the solution for different value of $\mathrm{N}$

This is a text of acknowledgements. Do not forget people who have assisted you on your work. Do not exaggerate with thanks. If your work has been paid by a Grant, mention the Grant name and number here.

\section{References}

[1] G. W. Bluman and S. Kumei, Symmetries and Differential Equations, Springer-Verlag, (1989).

[2] N. H. Ibragimov, Elementary Lie Group Analysis and Ordinary Differential Equations, John Wiley Sons, West Sussex, (1999).

[3] John. Starrett, "Solving Differential Equations by Symmetry Groups", American Mathematical Monthly, Vol.114, No.5, (2007), pp.778-792. 
[4] P. Glendinning, Stability instability an introduction to the theory of nonlinear differential equations, Cambridge England New York Cambridge University Press, (1987).

[5] G. Dahlquist and A. Bjorck, Numerical methods in scientific computing, Society for Industrial and Applied Mathematics (SIAM)Philadelphia, (2008).

[6] Grillakis. M, Shatah.J, Strauss.W, "Stability theory of solitary waves in the presence of symmetry", Journal of Functional Analysis, Vol.74 No.3, (1987), pp.160-197.

[7] Th. Simos, T. J. Kalvouridis.G, "plication of high-order Runge-Kutta methods in the magnetic-binary", An International Journal of Astronomy, Astrophysics and Space Science, Vol.147, No.2, (1988), pp.

[8] C. C. Christara and K. R. Jackson, "Scientific Computing by Numerical Methods", Encyclopaedia of Applied Physics, Vol.17, No.4, (1996), pp $1-79$

[9] Uri M.Ascher and Linda R.Petzold, Computer methods for ordinary Differential Equations and differential algebraic equations SIAM, (1998).

[10] E.S. Cheb-Terrab, L.G.S. Duarte, L.A.C.P "Computer algebra solving of second order ODEs using symmetry methods", Computer Physics Communications, Vol.108, No.3, (1988), pp. 90-114.

[11] C.W.Gear, Numerical Initial Value Problems in Ordinary Differential Equations Prentice-Hall Englewood-Cliffs, (1971).

[12] P.Phohomsiri and F.E Udwadia "Acceleration of Runge-Kutta integration schemes", Discrete Dynamics in Nature and Socity, Vol.2, No.5, (2004), pp. 307-314.

[13] Faranak Rabiei, Fudziah Ismail, Norazak, and Saeid Emadi "Numerical solution of second-order ordinary differential equations by improved Runge-Kutta Nystrom method", International Journal of Mathematical, Computational, Physical, Electrical and Computer Engineering, Vol.6, No.9, (2012), pp. 1372-1374. 